\title{
Essais cliniques exploratoires : quelles modalités de mise en œuvre, recommandations de mise en œuvre, champ d'application, cadre réglementaire?
}

\author{
Alain Francillon ${ }^{1}$, Gisèle Pickering ${ }^{2}$, Chantal Belorgey ${ }^{3}$ et les participants à la table ronde $n^{\circ} 2$ de Giens XXIV \\ 1 Novartis, Rueil-Malmaison, France \\ 2 Centre de Pharmacologie Clinique, CHU, Clermont-Ferrand, France \\ 3 Afssaps, Unité Essais Cliniques, Saint-Denis, France
}

Texte reçu le 15 octobre 2008 ; accepté le 20 novembre 2008

\begin{abstract}
Mots clés :
médicaments : sélection précoce des molécules; essais cliniques exploratoires ; mise en œuvre ; recommandations
\end{abstract}

Résumé - La table ronde s'est attachée à définir les essais cliniques exploratoires de médicaments et à proposer des recommandations relatives à leur mise en place en France : notamment les pré-requis non cliniques nécessaires, la qualité pharmaceutique du médicament expérimental et les conditions de réalisation de ces essais cliniques.

Pour ce faire, elle a tenu compte des recommandations existantes (américaines et belges notamment) et du projet de révision de la guideline ICH M3.

Les essais exploratoires sont des essais cliniques conduits précocement en phase I, avant l'escalade de dose et les études de tolérance et de sécurité d'emploi. Ces essais sont de fait une première administration de médicament à l'Homme mais n'ont pas de but thérapeutique ni diagnostique et ils ne visent pas la recherche de la dose maximale tolérée (MTD). Leur objectif est de répondre à des questions précises permettant de décider ou non de la poursuite du développement du médicament. Ils doivent inclure un nombre limité de patients ou sujets sains, exposés à une nouvelle substance à dose réduite et pendant une courte période.

Le projet de révision de M3 décrit 5 approches : 2 utilisant des microdoses, 3 des doses pharmacologiques.

Les principales recommandations du groupe peuvent se résumer ainsi :

- Un essai exploratoire est réalisé si c'est une étape justifiée, utile dans le développement d'un produit, et dans le respect des pré-requis.

- Le dossier pharmaceutique tient compte de la faible quantité de produit disponible, de la courte durée de l'administration du produit et des faibles doses utilisées. Les règles de bonnes pratiques de fabrication peuvent être adaptées.

- Le dossier non-clinique est également adapté à la phase de développement et aux particularités d'utilisation du produit. À cet égard, les recommandations de la révision de M3 sont commentées par le groupe.

- Le protocole doit comporter la réponse à un ensemble de questions spécifiquement pertinentes concernant cette phase d'administration, tout particulièrement sur les choix de la dose de départ, des paliers, et les règles d'arrêt d'un sujet et de l'essai.

- Les populations dites vulnérables de façon générale ne devraient pas être concernées par ce type d'essai, sauf exception dûment justifiée.

- Le groupe a estimé que les approches 3 à 5 ne devraient s'appliquer que pour un produit destiné à traiter une pathologie sévère ou rare, ou avec un besoin médical important.

- Une pré-soumission à l'Afssaps (Agence française de sécurité sanitaire des produits de santé) est conseillée notamment pour les approches à des doses pharmacologiques.

\footnotetext{
* Pour la liste des participants, voir en fin d'article.
} 
Il est aujourd'hui estimé que seulement 8 à $10 \%$ des nouvelles molécules entrant en phase I (5\% pour les molécules anticancéreuses) obtiendront une autorisation de mise sur le marché. ${ }^{[1,2]}$ Ce taux très faible de succès a été attribué à plusieurs causes : le manque de modèles animaux prédictifs de l'efficacité et de la toxicité de nouvelles molécules, le manque d'efficacité, la toxicité, une pharmacocinétique inappropriée, la complexité croissante des essais cliniques (impliquant cibles moléculaires et techniques avancées), le manque de biomarqueurs en particulier en cancérologie, les coûts élevés (en termes financier, de nombre de patients et de ressources professionnelles). Les grandes étapes du long processus de développement clinique (tolérance et sécurité d'emploi, efficacité, bénéfice thérapeutique) ont relativement peu changé au cours des 35 dernières années alors que de nouveaux outils sont accessibles. ${ }^{[3]}$ Il a été suggéré que le fait de pouvoir répondre très précocement à un certain nombre de questions critiques concernant une molécule (mécanisme d'action, cibles...) en tout début de développement, permettrait de diminuer le risque d'interrompre le projet en phase III. ${ }^{[4]}$ Une sélection précoce, notamment grâce aux « essais exploratoires », permet de se focaliser sur les molécules les plus prometteuses et de favoriser une mise à disposition rapide des innovations thérapeutiques.

\section{Introduction}

Peu d'essais de ce type ont été effectués pour l'instant en France. Il est important pour l'attractivité de notre pays que cette catégorie d'étude puisse être réalisée dans des centres français et dans des conditions garantissant la sécurité et la qualité de l'essai. L'objectif de la table ronde était donc de préciser le cadre de ces essais et d'élaborer des recommandations et propositions concrètes.

La table ronde s'est attachée à définir les essais cliniques exploratoires de médicaments et à proposer des recommandations relatives à leur mise en place en France, notamment concernant les pré-requis non cliniques nécessaires, la qualité pharmaceutique du médicament expérimental (ME) et les conditions de réalisation de ces essais cliniques. Pour ce faire, elle a tenu compte des recommandations existantes (américaines, de la Food and Drug Administration, ${ }^{[4]}$ de certains états membres ${ }^{[5,6]}$ et de la prochaine version de la guideline ICH M3 ${ }^{[7]}$ ).

\section{Comment définir un essai exploratoire?}

Afin d'éviter les confusions, le terme ambigu de «phase 0 » n'est pas à retenir et il est préférable de parler d'essai exploratoire. Les essais exploratoires sont des essais cliniques conduits précocement en phase I, avant les études classiques d'escalade de dose et les études de tolérance et de sécurité d'emploi. Ces essais sont de fait une première administration chez l'Homme et n'ont pas de but thérapeutique ou diagnostique. Ils ne visent pas la recherche de la dose maximale tolérée (MTD) comme en phase I classique mais à préciser précocement la pharmacocinétique et/ou la pharmacologie de la molécule. Leur objectif est de répondre à des questions précises permettant de décider ou non de la poursuite du développement du candidat médicament. Ils permettent par exemple d'éliminer une molécule très précocement en raison de propriétés pharmacodynamiques et/ou pharmacocinétiques médiocres (clairance rapide, biodisponibilité faible, absence d'effet sur la cible, etc.).

Ils incluent un nombre limité de patients ou de sujets sains, qui seront exposés à la nouvelle substance à dose réduite et pendant une courte période. Les essais exploratoires ne peuvent se substituer à la phase I classique. Un essai exploratoire n'est réalisé que si c'est une étape justifiée, utile dans le développement d'un produit, dans le respect des pré-requis et de la sécurité des individus qui se prêtent à la recherche.

La table ronde a proposé certaines limites à sa réflexion. Ainsi, les essais exploratoires ne concernent pas les produits qui présentent des facteurs de risque en raison de leur mode d'action, de la nature de la cible ou des caractéristiques des modèles animaux. ${ }^{[8]}$ De même, le cas des essais portant sur les produits de thérapie cellulaire et génique n'ont pas été pris en compte par la table ronde. Le recours aux essais exploratoires n'est justifié que si le rapport bénéfice/risque chez l'animal est élevé. Les populations dites vulnérables de façon générale ne devraient pas être concernées par ce type d'essais, sauf exception dûment justifiée.

En cancérologie la phase exploratoire est inappropriée lorsqu'il s'agit d'un cytotoxique à index thérapeutique étroit, que son utilisation est intraveineuse (IV) intermittente, qu'elle ne correspond pas à un essai de pharmacodynamie bien définie, ou quand enfin son mécanisme d'action est incertain.

\section{Les approches des essais exploratoires et la préclinique}

Le projet de révision de la guideline $\mathrm{ICH}$ M3 ${ }^{[7]}$ décrit 5 approches dont 2 utilisent des microdoses (objectif : receptor occupancy, biodistribution) et 3 des doses pharmacologiques, en administration unique ou répétée jusqu'à 14 jours [objectif : activité pharmacodynamique (PD) et/ou pharmacocinétique (PK, PK/PD)] (tableau I).

Le groupe a estimé que les approches 3 à 5 ne devraient s'appliquer que pour un produit destiné à traiter une pathologie sévère ou rare, ou avec un besoin médical important. 
Tableau I.

\begin{tabular}{|c|c|c|c|}
\hline & Approche & Préclinique & Commentaires du groupe \\
\hline 1 & $\begin{array}{l}\text { Microdose max } \\
100 \mu \mathrm{g}\end{array}$ & $\begin{array}{l}\text { Toxicologie dose unique étendue, } \\
1 \text { espèce }\end{array}$ & Approche déjà en place et utilisée. Génotoxicité non nécessaire \\
\hline 2 & $\begin{array}{l}\text { Microdose max } \\
5 \times 100 \mu g\end{array}$ & Toxicologie 7 jours, 1 espèce & $\begin{array}{l}\text { Étude de toxicité à réaliser par voie intraveineuse et voie d'administration } \\
\text { clinique ou seulement voie clinique. Génotoxicité : test d'Ames si approche } \\
\text { structure-activité par logiciel positive }\end{array}$ \\
\hline 3 & $\begin{array}{l}\text { Dose unique } \\
\text { pharmaco } \\
\text { logique }\end{array}$ & $\begin{array}{l}\text { Toxicologie dose unique étendue, } \\
2 \text { espèces génotoxicité }\end{array}$ & $\begin{array}{l}\text { Objectif PK, PD ou PK/PD en dose unique. La dose maximum } 1 / 2 \text { NOAEL } \\
\text { n'apparaît pas acceptable car trop élevée : ce serait une dose de phase I. }\end{array}$ \\
\hline 4 & $\begin{array}{l}\text { Dose unique ou } \\
\text { répétée jusqu'à } \\
14 \text { jours }\end{array}$ & $\begin{array}{l}\text { Toxicologie } 2 \text { semaines, } \\
2 \text { espèces Génotoxicité } \\
\text { Pharmacologie de sécurité } \\
\text { PK }\end{array}$ & $\begin{array}{l}\text { Cinétique animale détaillée indispensable pour pouvoir extrapoler la PK } \\
\text { clinique d'après la } \mathrm{PK} \text { chez les animaux. } \\
\text { Marge de sécurité maximum à atteindre à définir selon les résultats de } \\
\text { pharmacologie de sécurité et de toxicologie }\end{array}$ \\
\hline 5 & & & $\begin{array}{l}\text { Ce modèle implique une activité pharmacologique anticipée pour une } \\
\text { dose éloignée de la dose toxique }\end{array}$ \\
\hline
\end{tabular}

PK : pharmacocinétique ; PD : pharmacodynamique ; NOAEL : No Observed Adverse Effect Level, dose sans effet toxique chez l'animal ; Marge de sécurité : rapport exposition chez l'animal à la NOAEL/exposition chez l'homme à la dose clinique, l'exposition est mesurée par AUCss ou Cmaxss ; AUC : Area Under the Curve, surface sous la courbe des concentrations plasmatiques; AUCss : AUC à l'état d'équilibre ; Cmaxss : concentration plasmatique maximum à l'état d'équilibre.

\section{1. Recommandations relatives à la Qualité pharmaceutique du médicament expérimental (hors produits biologiques)}

Le cahier des charges porte sur un produit synthétisé en quantité faible, une dose administrée infime et une administration de courte durée, mais avec une substance active caractérisée et une formulation développée a minima. Pour les approches 1 et 2 (microdoses), la substance active doit être caractérisée à partir des données de recherche, le schéma de synthèse décrit à l'échelle «laboratoire». Une analyse de risque théorique sur l'exposition aux impuretés doit être faite notamment par rapport à la concentration administrée, enfin les méthodes de contrôle de la substance active doivent être adaptées avec une description des aspects critiques de la stabilité. Pour le produit fini, le cas particulier d'un produit stérile nécessitera une approche spécifique (analyse de la compatibilité contenant-contenu, description des aspects critiques de solubilisation, cristallisation, validation de la stérilisation).

Pour les approches 3 à 5 , les pré-requis de phase I devront s'appliquer. ${ }^{[9]}$

En terme de Bonnes Pratiques de Fabrication, il apparaît possible de développer des pré-requis Qualité plus flexibles pour les essais exploratoires des approches 1 et 2 ; pour ces essais de microdose, le groupe propose de se référer aux Bonnes Pratiques de Préparation élaborées par l'Afssaps. ${ }^{[10]}$

\subsection{Radiopharmaceutiques}

Les remarques précédentes s'appliquent au produit froid. Pour le produit radiomarqué, la réglementation française demande des autorisations administratives spécifiques. Des recommandations ont été proposées au niveau européen. ${ }^{[1]}$

\subsection{Pré-requis non cliniques}

Le groupe a revu en détail et commenté les pré-requis non cliniques (toxicité/génotoxicité/pharmacologie de sécurité) proposés par le projet de révision de la guideline ICH M3 ${ }^{[7]}$ pour chaque approche. Il a été rappelé que les études de toxicologie (toxicocinétique inclus) et de pharmacologie de sécurité doivent suivre les Bonnes Pratiques de Laboratoire. L'essentiel des commentaires sont décrits dans le tableau I.

L'essai clinique doses uniques doit être terminé avant de procéder à l'essai clinique doses répétées.

\section{Réalisation de l'essai clinique}

Ces essais doivent être menés dans les mêmes conditions que les essais de première administration à l'homme, conformément à la recommandation de l'EMEA (European Medicines Agency) et de l'Afssaps, ${ }^{[8,12]}$ et notamment dans des sites disposant d'une autorisation de lieux. Certains essais peuvent nécessiter une capacité technique adaptée, une qualification particulière de l'investigateur, l'implication d'un pharmacologue.

Si l'essai est multicentrique, il faudra le justifier et organiser la coordination et la transmission en temps réel des informations entre centres.

En outre, il convient de décrire et justifier dans le protocole la fréquence et les modalités d'administration du médicament (intervalles entre les sujets, périodes de « wash-out», nombre de sujets 
administrés le même jour), de ne pas administrer simultanément plusieurs ME, de ne pas administrer successivement plusieurs ME chez le même patient/sujet dans le même essai sauf justification au cas par cas.

Il conviendra de justifier le choix des doses administrées et leur répétition éventuelle. Il pourrait être proposé que la dose devrait, dans tous les cas, déterminer une exposition inférieure à celle rapportée au 1/10 ${ }^{\mathrm{e}}$ de la NOAEL (No Observed Adverse Effect Level) ou de la MABEL (Minimum Anticipated Biological Effect Level) dans le cas des produits biologiques.

Le document d'information et de consentement du sujet sain ou malade est comparable à celui des essais phase I, précisant notamment l'absence d'objectif thérapeutique, l'absence de bénéfice individuel, ${ }^{[13]}$ et le niveau de risque évalué en fonction de l'approche 1 à 5 .

Les critères d'arrêt de l'essai clinique sont définis : l'arrêt est prévu dès que l'objectif prédéfini dans le protocole est atteint ou s'il apparaît que l'on ne pourra pas l'atteindre. Pour les microdoses (approche 1 ou 2), aucune manifestation d'intolérance n'est acceptable. Pour les autres approches (3 à 5) les critères d'arrêt seront définis dans le protocole, y compris toute manifestation clinique ou paraclinique différente de l'objectif poursuivi. ${ }^{[8,12]}$

\section{1. Validité des critères de jugement et des méthodes utilisés dans ces essais}

Il s'agit d'un pré-requis indispensable. D'une manière générale, les méthodes analytiques du critère de jugement doivent être pertinentes, avec utilisation de critères validés (en termes de répétabilité, sensibilité, variabilité, robustesse). Plusieurs auteurs du National Cancer Institute ${ }^{[3,14,15]}$ recommandent ainsi l'utilisation de deux tests différents pour évaluer le mécanisme d'action d'un produit sur une cible. Cette recommandation semblent raisonnables, notamment lorsque la relation PK/PD n'a pas encore été recherchée ou en l'absence de relation établie.

Ce solide critère de jugement (essai PD) recevra ici sa validation première chez l'homme, et pourra ainsi être utilisé dans les études ultérieures pour reconnaître la modulation de la cible. Cette information précoce PK/PD permettra dans le cadre de la cancérologie d'optimiser ultérieurement les techniques d'escalade de doses de la phase I.

\subsection{Populations}

Le choix d'un sujet sain ou d'un patient sera explicité. ${ }^{[8,12]}$ Le nombre de sujets/patients sera limité. D’une façon générale, les essais exploratoires ne devraient pas s'appliquer en pédiatrie sauf exception dûment justifiée. De même, ils ne devraient pas s'appliquer aux autres populations dites vulnérables.

\subsection{Préparation de la demande d'autorisation de l'essai clinique}

La qualification de l'essai en étude exploratoire devra clairement être mentionnée dans le protocole, le courrier et le formulaire de demande d'autorisation. Une pré-soumission de l'essai à l'Afssaps est conseillée notamment pour les approches 3 à 5 ( $c f$. procédure de pré soumission sur le site internet de l'Afssaps). Pour le CPP (Comité de Protection des Personnes), la discussion CPP-promoteur-investigateur est encouragée.

\section{Conclusions}

Les études cliniques exploratoires visent à répondre très précocement aux questions critiques concernant une molécule avant de commencer un projet complet de développement.

Les essais exploratoires sont possibles en France. L'Afssaps recommande une pré-soumission notamment pour les essais de type 3 à 5 (recherche d'activité pharmacologique).

Le groupe a proposé des conditions d'applications de ICH M3, suggérant ainsi une ouverture encadrée vers ces essais cliniques.

\section{Participants}

Michel Abiteboul (Quintiles), Bernard Bégaud (Département de Pharmacologie, CHU Pellegrin Carreire, Bordeaux), Jehan-Michel Behier (MSD Chibret), Chantal Belorgey (Afssaps), Bénédicte Chauvin (Novartis), Antoine Cournot (Thérapharm), Rémy Defrance (Pfizer), Jean-Paul Demarez (Pierre Fabre Médicaments), Élisabeth Devilliers (Fédération Hospitalière de France), Vincent Diebolt (GIP CeNGEPS), Bertrand Diquet (Pharmacologie, CHU Larrey, Angers), Yves Donazzolo (OPTIMED Clinical Research), Muriel Echemann (Afssaps), Alain Francillon (Novartis), Christian Funck-Brentano (Pharmacologie, Faculté de Médecine Pitié Salpétrière, Paris), Patrick Genissel (IRI Servier), Nadine Houedé (Institut Bergonié, Bordeaux), Jean-Marc Husson (European Diploma in Pharmaceutical Medicine/Eudipharm, Paris), Philippe Lefevre (Afssaps), Isabelle Macquin-Mavier (Pharmacologie Clinique, Hôpital Henri Mondor, Créteil), Brigitte Marchenay (Roche), Christine Marey (Science Union, les Laboratoires Servier), Laurence Negres-Pages (LN Pharma), Gisèle Pickering (Pharmacologie Clinique, CHU Clermont Ferrand, Clermont-Ferrand), Séverine Pitel (Pharmacologie, CHU Timone, Marseille), Valérie Plattner (Direction de la Recherche Clinique et de l'Innovation, CHU Lyon, Lyon), Christine Roy (Sanofi Aventis Research and Developpment), Michel Sibille (Centre Hospitalier Lyon Sud, Pierre Bénite), 
Nicolas Simon (Faculté de Médecine, Marseille), Claire Thalamas (CHU de Toulouse, Hôpital Purpan, Toulouse), Dominique Tremblay (Afssaps), Tony Waegemans (LFB).

\section{Références}

1. Marchetti S, Schellens JHM. The impact of FDA and EMEA guidelines on drug development in relation to Phase 0 trials. Br J Cancer 2007; 97: 57781

2. Concept paper on the development of a CHMP guideline on the non-clinical requirements to support early phase I clinical trials with pharmaceutical compounds. EMEA/CHMP/SWP/91850/2006. http://www. emea. europa.eu

3. Doroshow JH, Parchment RE. Oncologic phase 0 trials incorporating clinical pharmacodynamics: from concept to patient. Clin Cancer Res 2008; 14(12): 3658-63

4. Guidance for industry, investigators and reviewers on exploratory IND studies. FDA, January 2006. http://www. fda.gov

5. Guidance to the conduct of exploratory trials in Belgium. Working Document Federal Agency for Medicines and Health Products. http://www. afmps. be

6. Report of the international expert meeting on Exploratory clinical trial application and microdosing. 15 Sept 2007. BfArM (Germany)

7. ICH Topic M3 (R2) Note for guidance on non-clinical safety studies for the conduct of human clinical trials and marketing authorization for pharmaceuticals compounds. CHMP/ICH//286/95 July 2008 (draft in consultation)
8. Guidance on strategies to identify and mitigate risks for First-inHuman clinical trials with investigational medicinal products. EMEA/CHMP/SWP/28367/07 (19 July 2007). http://www. emea. europa.eu/pdfs/human/swp/2836707enfin.pdf

9. Guideline on the requirements to the chemical and pharmaceutical quality documentation concerning investigational medicinal products in clinical trials. EMEA, 2006. http://www. emea. europa. eu

10. Bonnes pratiques de fabrication, Afssaps, décembre 2007. http://www. afssaps.fr

11. Verbruggen A, Coenen H, Deverre JR, et al. Guideline to regulations for radiopharmaceuticals in early phase clinical trials in the EU. Eur J Nucl Med Mol Imaging 2008; 35: 2144-51

12. Essais de première administration à l'Homme, Afssaps, juillet 2006. http:// Www. afssaps.fr

13. Guttierez M, Collyar D. Patient perspective on phase 0 clinical trials. Clin Cancer Res 2008; 14(12): 3689-91

14. Calvert AH, Plummer R. The development of phase I cancer trial methodologies: the use of pharmacokinetic and pharmacodynamic end points sets the scene for phase 0 cancer clinical trials. Clin Cancer Res 2008; 14(12): 3664-9

15. Murgo AJ, Kummar S, Rubinstein L, et al. Designing phase 0 cancer clinical trials. Clin Cancer Res 2008; 14(12): 3675-82

Correspondance et offprints : Alain Francillon, Novartis, 2-4 rue Lionel Terray, BP 308, 92506 Rueil-Malmaison Cedex, France.

E-mail : alain.francillon@novartis.com 